\title{
Tolerance Analysis for Clock Mechanism Structural Parameters
}

\author{
Qing-sen Chen \\ Science and Technology on Electromechanical \\ Dynamic Control Laboratory \\ Beijing Institute of Technology \\ Beijing China \\ 1990chs@163.com
}

Rong-chang Song

Science and Technology on Electromechanical

Dynamic Control Laboratory

Beijing Institute of Technology

Beijing China

bit_src@bit.edu.cn

\author{
Ping Guan \\ Science and Technology on Electromechanical \\ Dynamic Control Laboratory \\ Beijing Institute of Technology \\ Beijing China \\ yiranhenzhun@163.com \\ Hai-bin Cui \\ The Huaihai Industrial Group Limited Corporation \\ Changzhi China \\ cuihaibin@163.com
}

\begin{abstract}
In a given clock mechanism, the tolerances of its structural parameters often cast profound influences to the accuracy of the timing output. In this paper, the tolerances of the clock mechanism parameters are studied to explore its influence on timing. Through 3D modelling of the proposed clock mechanism along with related multi-rigid-body dynamics simulations, full factorial experimental design method is used to investigate the impacts of structural parameters onto the timing contribution. The results of experimental design illustrate that the center distance between escape wheel and pendulum, and the diameter of escape wheel addendum have a higher influence on the timing of clock mechanism. Based on the probability distributions of the clock mechanism structural parameters, Monte Carlo simulation is applied to obtain the probability distributions of timing. The simulation results suggest that the timing follows the pattern of a normal distribution and most of its values are in the vicinity of the average.
\end{abstract}

Keywords- clock mechanism; tolerance analysis; contribution analysis; full factorial experimental design; Monte Carlo simulation

\section{INTRODUCTION}

There are a number of uncertain factors, such as manufacturing errors, elastic deformation causing variations in mechanism performance. Then many manufactural products fail meet the design requirements and their robustness is poor, thus some tolerances of the products should be analysed. The tolerance analysis methods include worst-case tolerance analysis and statistical tolerance analysis [1]. Worst-case tolerance analysis considers the worst possible combinations of individual tolerances, lead to excessively tight part tolerance and high production costs. On the contrary, statistical tolerance analysis is a more practical and economical way on setting the tolerances so as to ensure a desired yield. Some scholars use these methods to investigate the products tolerances. For example, Dantan J Y [2] applied worse-case method based on quantified and statistical method based on Monte Carlo simulation to analyze tolerances; Doltsinis I [3] used optimization methods to design structures parameters including sensitivity analysis and contribution analysis; the robustness optimization had been applied to analyze tolerances by Zang C [4], Xianzhen Huang [5], ZHOU Kai [6], and so on. Experimental design method was used to analyze the analysis of tolerances. Statistical tolerance analysis is applied to study the tolerances of the clock mechanism parameters in this paper.

Clock mechanism is often used as a timing mechanism in fuze safety system, and it has many advantages such as good anti-jamming capacity, strong environment adaptability. The theoretical calculation of clock mechanism in a certain security system meets the design requirements, but the experimental test results of clock mechanism prototype fail meet the requirements. So, many scholars [7-9] use virtual prototype simulation methods to find the reasons. It was found that the structural parameters of clock mechanism, including the arc radius and diameter of escape wheel addendum and the center distance of escape wheel and pendulum, had impacts on timing accuracy. These parameters led to a disperse distribution of timing and poor robustness for the design requirements.

Dr. Song [10] had analyzed their effects on the contribution of timing. But he only changed one of several structural parameters without changing the others and for each parameter he merely conducted seven groups of simulation. Therefore the lack of evidence cases some limitations in this thesis: these parameters, in fact, interact with each other concurrently and the sample number is insufficient.

In this paper, full factorial experimental design method is applied to analyze the contribution of parameters tolerances, which varies these parameters simultaneously 
and has an adequate volume of simulation times to ensure its accuracy. Furthermore, according to the statistics of actual machining dimension, the probability distributions of these parameters are achieved. Then Monte Carlo simulation is conducted to evaluate the probability distributions of timing when these parameters follow statistical distribution within their tolerance.

\section{THE MODEL OF CLOCK MECHANISM}

Clock mechanism [11] usually consists of three parts: driving gear, gear train, and pendulum wheel. A threedimensional model of clock mechanism is shown as Fig.1, which is driven by double-slider mechanism. The rack of the upper slide is meshed with the driving gear. It works as follows. When the projectile is launched by a high acceleration, the double slider will be subjected to acceleration loads. Then the rack of the upper slide drives the driving gear to make clock mechanism movement. Under the control of clock mechanism, the arming time of fuze can be controlled effectively.

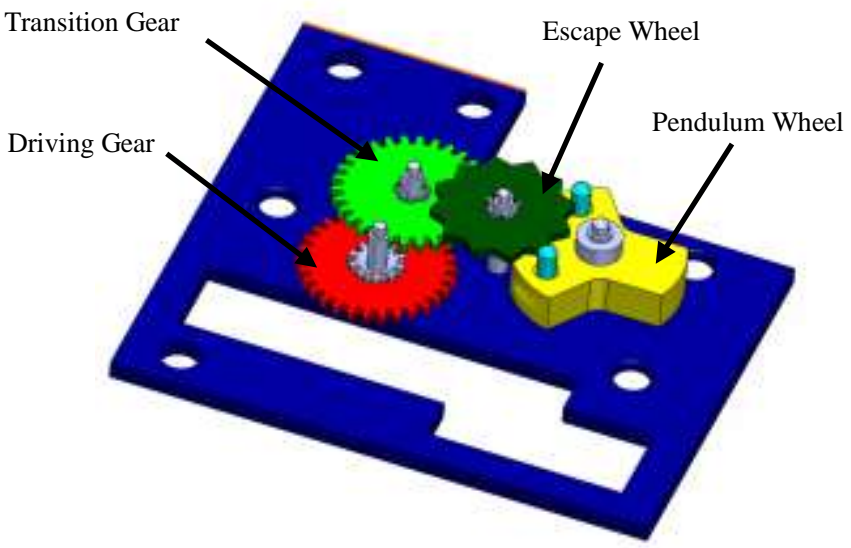

Figure 1. The Model of Clock Mechanism

Due to the part deformations in clock mechanism are small during operation and have little effect on timing, the clock mechanism is regarded as multi-rigid-body systems. The 3D model of mechanical system can be built accurately and it can represent the structural parameters tolerances and their distribution. Based on 3D model and dynamics simulation method, the dynamics simulation of clock mechanism is carried out. Thus, the main process is as follows. First of all, the 3D model of clock mechanism is built in the UG NX software. Then the diameter of escape wheel addendum, the center distance of escape wheel and pendulum, the center distance of pendulum and pendulum pin and the center distance of two pendulum pin are appointed as the parameters, so the actual processing size can be simulated through altering these parameters values. Finally, multi-rigid body dynamics simulation is built in the ADAMS software. In order to change parameters values and make simulation automatic, the Isight software is used to integrate UG and ADAMS software. Equivalent force-moment is applied to the escape wheel to reduce simulation time and improve computational efficiency.

The parameters needed to be analyzed in this paper are listed in Table I and shown as Fig .2. The variable Time is represent as the timing of clock mechanism.
TABle I The Parameters of Clock Mechanism

\begin{tabular}{|c|c|c|c|c|c|}
\hline Parameter & D & Lx & Ly & Px & Py \\
\hline $\begin{array}{c}\text { Nominal } \\
\text { Dimension(mm) }\end{array}$ & 7.480 & 5.23 & 0.00 & 1.900 & 5.00 \\
\hline Tolerance $(\mathrm{mm})$ & $\begin{array}{c}0.000 \\
-0.036\end{array}$ & \pm 0.03 & \pm 0.03 & \pm 0.015 & \pm 0.02 \\
\hline Size Range $(\mathrm{mm})$ & $\begin{array}{c}7.444- \\
7.480\end{array}$ & $\begin{array}{c}5.20- \\
5.26\end{array}$ & $\begin{array}{c}-0.03- \\
0.03\end{array}$ & $\begin{array}{c}1.885- \\
1.915\end{array}$ & $\begin{array}{c}4.98- \\
5.02\end{array}$ \\
\hline
\end{tabular}

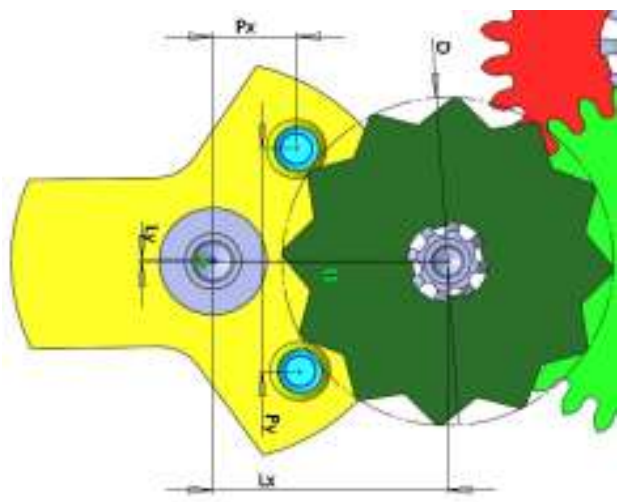

Figure 2. The Meanings of Parameters of Clock Mechanism

Where $\mathrm{D}$ is the diameter of escape wheel addendum; $\mathrm{Lx}$ is the $\mathrm{x}$ direction distance of the center of escape wheel and pendulum; Ly is the y direction distance of the center of escape wheel and pendulum; $\mathrm{Px}$ is the direction distance of pendulum and pendulum pin; Py is the center distance of two pendulum pin.

\section{SIMULATION OF CLOCK MECHANISM}

\section{A. The Verification of Model}

The simulation of clock mechanism model is carried out when its initial parameters are nominal dimensions. The rotation angle of escape wheel and the rotation angle of pendulum wheel are shown as Fig .3 and Fig .4.

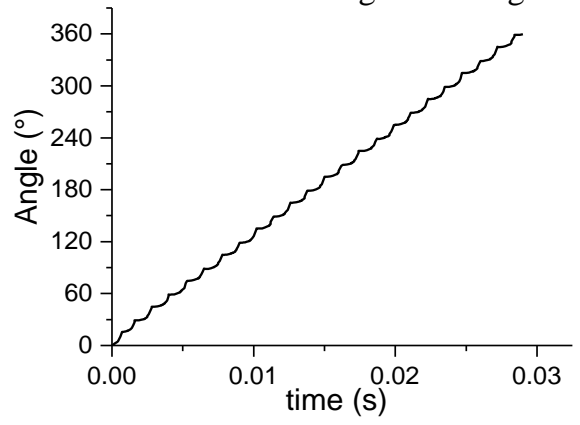

Figure 3. The Rotation Angle of Escape Wheel

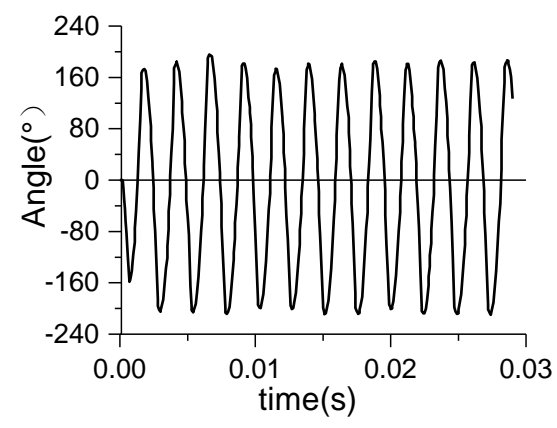

Figure 4. The Rotation Angle of Pendulum Wheel 
The results show that established model is correct and the rotation angle of escape wheel is increased in a staged way. Furthermore, the rotation angle of pendulum wheel is followed a cyclical pattern. In this paper, the timing is the time when the rotation angle of pendulum wheel is 360 degree. Then the relationship between timing and structural parameters is discussed in the following sections.

\section{B. Contribution Analysis of Parameters}

The dimension contributions are the degree that the dimensions tolerances affect the design function [12]. Its values are mainly relevant to the dimension tolerances. If the product do not meet the require performance, the tolerances having a bigger contribution are always decreased to meet these requirements. Hence, the dimension contributions are one of the indexes which the product tolerances are adjusted.

In order to analyze the influence of structural parameters on timing of clock mechanism, design of experiment (DOE) is used to acquire the contribution. DOE methods include orthogonal experiments design, full factorial experimental design, and so on. Full factorial experimental design is utilized to develop better experimental results and the whole process is shown as Fig .5. In this part, each parameter selects 4 levels which are listed in Table II, therefore there are 1024 simulation times in total. The simulation result is shown as Fig .6.

TABle II . THe LeVels of EACH PARAMETER

\begin{tabular}{|c|c|c|c|c|c|}
\hline Parameters & D & Lx & Ly & Px & Py \\
\hline & 7.444 & 5.20 & -0.03 & 1.885 & 4.980 \\
& 7.456 & 5.22 & -0.01 & 1.895 & 4.993 \\
Levels(mm) & 7.468 & 5.24 & 0.01 & 1.905 & 5.007 \\
& 7.480 & 5.26 & 0.03 & 1.915 & 5.020 \\
\hline
\end{tabular}

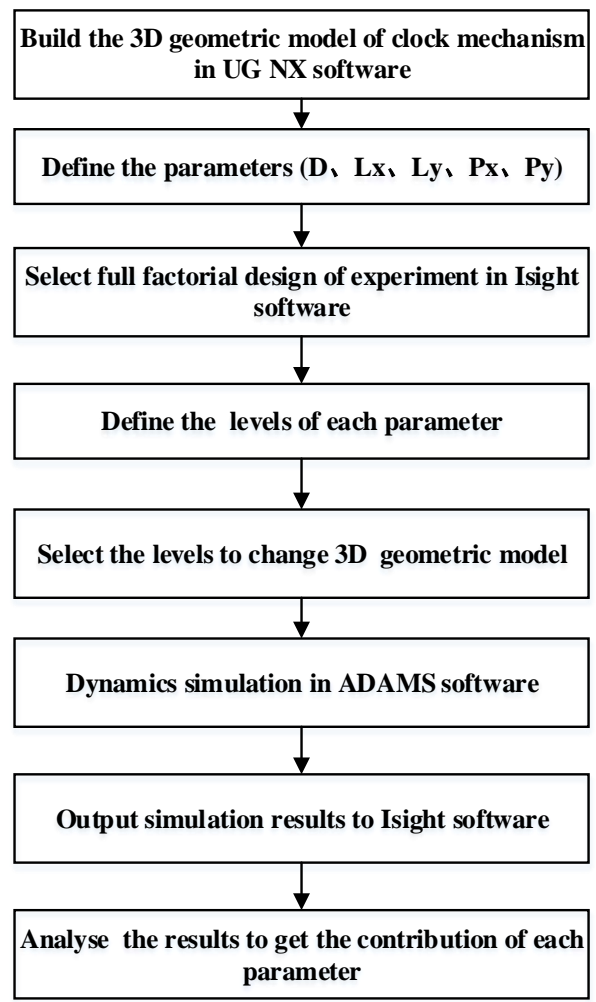

Figure 5. The Flow of Contribution Analysis

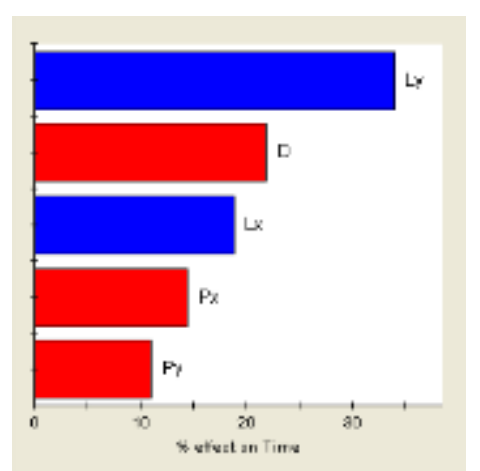

Figure 6. The Result of Contribution Analysis

The result shows there is a positive correlation between Time and variables of Ly and Lx, and there is a negative correlation between Time and variables of D, Px and Py. The Ly variable has a greater influence on Time than other variables. Thus the Ly value should be strictly controlled in the design of clock mechanism to reduce its effect of timing.

\section{Timing Distribution of Clock Mechanism}

In the actual parts machining process, the dimensions of parts will be distributed in some kind of regularity. Monte Carlo simulation is exploited to study the distribution of timing when the parameters are random values in their distribution, and the whole process is shown as Fig .7. Descriptive sampling method is used in Monte Carlo simulation and the sample number is 1500 to ensure the accuracy of sampling. Through the study of statistics of the actual machining dimension of each parameter, these parameters fit the normal distribution. Hence the parameter values are chosen from Fig .8 (a) to Fig .8 (e) and the simulation result is shown in Fig .8 (f) and Table III.

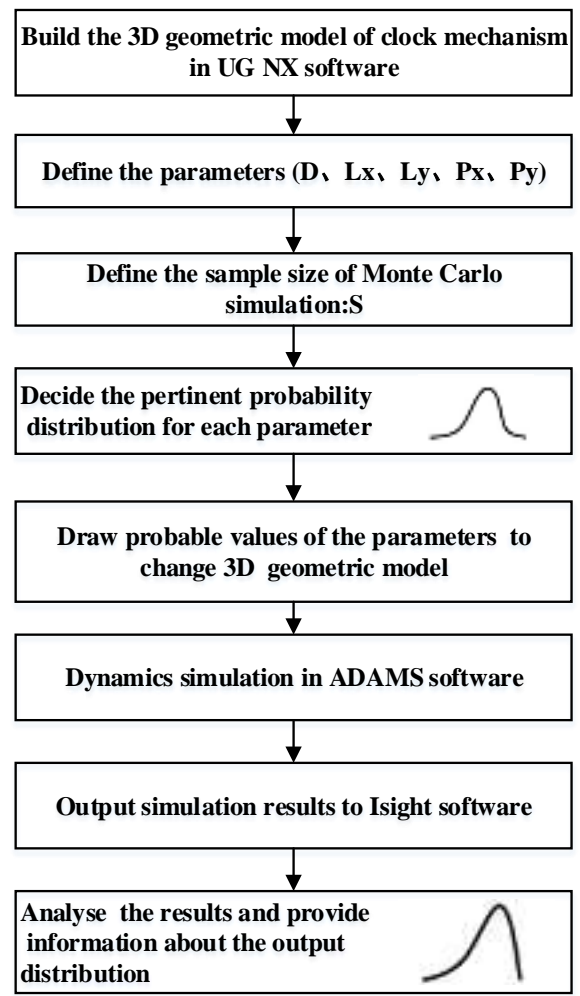

Figure 7. The Flow of Monte Carlo Simulation 


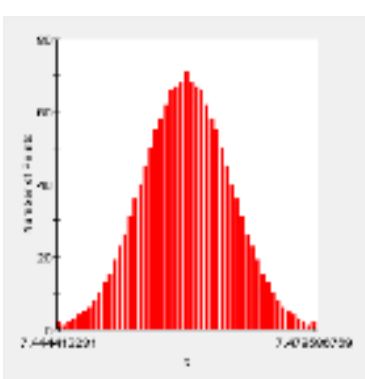

(a)

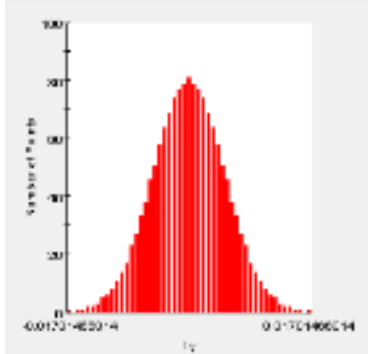

(c)

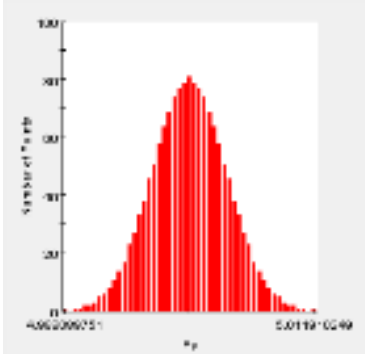

(e)

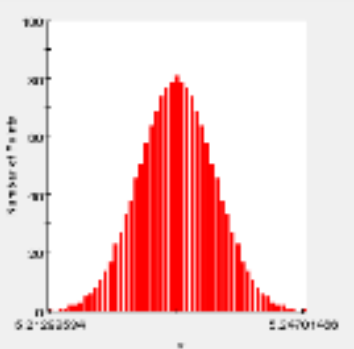

(b)

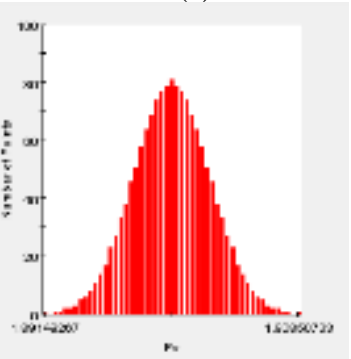

(d)

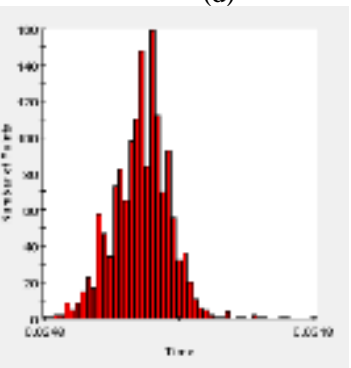

(f)
Figure 8. Probability Distributions of Parameters and Time

TABle III . The Result of Monte Carlo Simulation

\begin{tabular}{|c|c|c|c|c|}
\hline & $\begin{array}{c}\text { Mean } \\
\text { Value }\end{array}$ & $\begin{array}{c}\text { Standard } \\
\text { Deviation }\end{array}$ & $\begin{array}{c}\text { Upper } \\
\text { Limit } \\
\text { Value }\end{array}$ & $\begin{array}{c}\text { Lower } \\
\text { Limit } \\
\text { Value }\end{array}$ \\
\hline Time(s) & 0.027332 & $7.4906 \mathrm{E}-4$ & 0.0318 & 0.0248 \\
\hline
\end{tabular}

According to Fig .8 (f) and Table III, the timing distributions of clock mechanism is normal distribution. The mean value of Time is 0.027332 and most of its values are near to its mean value. As a result, the timing of clock mechanism can be adjusted to around the mean value to stabilize the distribution.

\section{CONCLUSION}

In this paper, computer 3D modeling and virtual prototype simulation are used to analyze the structural parameters of clock mechanism which have a gigantic influence on timing accuracy. The deformations of clock mechanism are ignored and the clock mechanism is regard as multi-body systems. Then contribution analysis by full factorial experimental design and distribution analysis by Monte Carlo simulation are applied to study their relationship. It is found that the center distance of escape wheel and pendulum and the diameter of escape wheel addendum have the greatest influence on timing. The timing of clock mechanism fits normal distribution. Based on the results, the structural parameters of clock mechanism can be optimized in the future. It would be foreseen that the optimized parameters which would make the timing approach the mean value have to most potential to improve the robustness of clock mechanism.

\section{REFERENCES}

[1] Wu F, Dantan J Y, Etienne A, Ali Siadat and Patrick Martin, "Improved algorithm for tolerance allocation based on Monte Carlo simulation and discrete optimization," Computers \& Industrial Engineering, vol. 56, Sep. 2009, pp. 1402-1413.

[2] Dantan J Y and Qureshi A J. "Worst-case and statistical tolerance analysis based on quantified constraint satisfaction problems and Monte Carlo simulation," Computer-Aided Design,vol. 41, Nov. 2009, pp. 1-12.

[3] Doltsinis I and Kang Z. "Robust design of structures using optimization methods," Computer Methods in Applied Mechanics and Engineering, vol. 193, Dec. 2004, pp. 2221-2237.

[4] Zang C, Friswell M I and Mottershead J E, "A review of robust optimal design and its application in dynamics," Computers \& Structures, vol. 83, Oct. 2005, pp.315-326.

[5] Huang $\mathrm{X}$ and Zhang $\mathrm{Y}$, "Robust tolerance design for function generation mechanisms with joint clearances," Mechanism and machine theory, vol. 45, May 2010,pp. 1286-1297.

[6] ZHOU Kai, CAI Ying, ZHANG Zhen-jia and LI Xiang-yuan. "Application of theory of robust design in tolerance analysis," Transactions of Bering Institute of Technology, vol. 23, Oct. 2003,pp. 557-560.

[7] Wang Lixin and Huang Wenliang, "Dynamics of straight-sided verge runaway escapement based on virtual prototype," Journal of Detection \&Control, vol. 27,Mar. 2005, pp. 27-30.

[8] Deng Hongbin and Liu Mingjie, "Design of timing device in clock mechanism with unturned escapement," Transactions of Bering Institute of Technology, vol. 19, Dec. 1999, pp. 51-55.

[9] Shang Yaling and Ma Baohua, "The Study on the vibration cycle of a pin runaway escapement," Journal of Detection \& Control, vol. 21, Jan. 1999, pp. 40-45.

[10] SONG Rong-chang, LI Ping, TAN Hui-min and SHI Song-tao, "Timing Dispersion of Clock Mechanism with Parameter of Escapement Mechanism," Transactions of Beijing Institute of Technology, vol. 30, Mar. 2010, 30(3):263-265.

[11] Anon. Engeering design handbook for fuzes, Beijing:The People's Liberation Army General Armament Department,2003.

[12] Wu Shaotong and Yang Jiangxin, Computer aided tolerance optimization design, Hangzhou: Zhejiang University press, 1999 